\title{
THE IMPORTANCE OF NON-INVASIVE REGIONAL BRAIN OXIMETRY IN PREVENTING POSTOPERATIVE COGNITIVE DYSFUNCTION DURING LAPAROSCOPIC CHOLECYSTECTOMY IN ELDERLY AND SENILE PATIENTS
}

\author{
Olena Bielykh \\ Department of Anesthesiology, Pediatric Anesthesiology and Intensive Care \\ Kharkiv Medical Academy of Postgraduate Education \\ 58 Amosova str., Kharkiv, Ukraine, 61176 \\ Bielykholena@gmail.com
}

\begin{abstract}
The adverse effect of general anesthesia in elderly patients during surgery requires monitoring of functions and processes to identify their dangerous abnormalities to prevent complications.

The aim of the study. To establish the effectiveness of non-invasive regional brain oximetry with $\mathrm{rSO}_{2}$ determination during anesthetic support in laparoscopic cholecystectomy (LCE) to prevent postoperative cognitive dysfunction in elderly and senile patients.

Material and methods. 84 elderly and senile patients with diagnosed gallstone disease, acute cholecystitis, who underwent LCE with sevofluran inhalational anesthesia and total intravenous anesthesia with propofol were examined. $\mathrm{rSO} 2$ was monitored. Cognitive functions were assessed using neuropsychological scales and the MMSE mental state examination scale.

Results. $\mathrm{rSO}_{2}$ was found to be significantly decreased during the carbon dioxide insufflation as compared to before premedication: in LH (left hemisphere) - by $7.0 \%$, in RH (right hemisphere) - by $6.9 \%$ (Group I); in LH - by $7.4 \%$, in RH - by $7.5 \%$ (Group II). $\mathrm{rSO}_{2}$ was significantly increased during the surgery, particularly in the middle of the operation, as compared to before premedication: in LH - by $14.66 \%$, in RH - by $13.94 \%$ (Group I); in LH - by $11.60 \%$, in RH - by $11.53 \%$ (Group II). The day following the surgery, cognitive functions significantly decreased by $8.7 \%$ on the Luria's test, by $6.0 \%$ on the MMSE test (Group I); in Group II - by $10.1 \%$ and $6.3 \%$, respectively, as compared to before premedication. On Day 5 after the surgery, cognitive functions decreased by $2.7 \%$ on the Luria's test, by $0.35 \%$ on the MMSE test (Group I); in Group II - by $2.7 \%$ and $0.35 \%$, respectively, as compared to before premedication.

Conclusions. A decrease in $\mathrm{rSO}_{2}$ can occur during LCE in the Trendelenburg position, despite the fact that other intraoperative indicators remain stable, which allows it to remain unrecognized. $\mathrm{rSO}_{2}$ monitoring in the perioperative period contributes to alertness and timely measures to prevent postoperative cognitive dysfunction.

Keywords: regional oximetry, cerebral hemoglobin oxygen saturation, $\mathrm{rSO}_{2}$, postoperative cognitive dysfunction, elderly and senile persons, laparoscopic cholecystectomy.
\end{abstract}

DOI: $10.21303 / 2504-5679.2020 .001351$

\section{Introduction}

The modern concept of anesthetic support aims not only to provide adequate pain relief, but also to control all the patient's vital functions during the operation. It should be noted that in the last decade, the concern about the adverse effect of general anesthesia on the elderly patients' brain has been growing, since these surgical patients often have postoperative cognitive dysfunction, which in turn can cause an increase in morbidity and mortality [1]. Postoperative cognitive dysfunction (POCD) is a disorder that develops in the early postoperative period, persists for several days or weeks, more rarely - for months, and is clinically manifested by memory disorders, difficulties in concentration and long-term focus of attention, as well as higher cortical function impairment such as thinking, speech, etc. The patient has problems with learning, mental performance decreases, mood worsens, and depression occurs. All of this can lead to a decrease in the quality of life. Therefore, one of the most important tasks of modern anesthesiology is to objectify the general anesthesia monitoring, which provides for the control of functions and processes, identifying their dangerous abnormalities to prevent complications during anesthesia. Not only the cardiovascular and respiratory system functions should be monitored, but also the state of the central nervous system (CNS), in particular the functional state of the brain. According 
to statistics, the most frequent complications in the process of anesthesia are various hypoxic conditions of the brain, which often cause POCD, especially in elderly and senile patients [2, 3].

Since the main tasks of neuromonitoring during anesthesia are to maintain and preserve the physiological body functions and provide optimal conditions for their improvement, the method of cerebral oximetry reflects the balance between the delivery and consumption of oxygen, which makes it possible to visualize the oxygen status of the brain and assess the oxygen saturation of tissues [4]. Today, the interest in cerebral oximetry has significantly increased due to non-invasiveness of the method, its highest level of safety, as well as technical achievements that have made this method more accurate and informative [5].

Now the problem of safety of anesthesia during surgical interventions in the elderly and senile patients does not lose its relevance. During general anesthesia, anesthetists have several tools to monitor the state of the brain. One of the methods to ensure the safety of anesthesia and prevent hypoxia episodes during anesthesia is to monitor the functional saturation of cerebral hemoglobin with oxygen $\left(\mathrm{rSO}_{2}\right)$. The main purpose of the non-invasive cerebral oximetry monitoring method is to measure regional cerebral oxygen saturation $\mathrm{rSO}_{2}$ using near-infrared spectroscopy [6].

An important risk factor for POCD is considered to be elderly and senile age, which is associated with a natural decline in cognitive functions and various concomitant diseases - hypertension, atherosclerosis, ischemic disease, thrombosis, strokes, etc. [7].

Cerebral oximetry is widely used in neonatology, pediatrics, thoracic, vascular, cardiac surgery, and neurology $[8,9]$. However, it remains unclear whether non-invasive regional brain oximetry is a reliable monitoring method for laparoscopic cholecystectomy to prevent POCD in elderly and senile population.

The aim of the work. To establish the effectiveness of non-invasive regional brain oximetry with $\mathrm{rSO}_{2}$ determination during anesthetic support in laparoscopic cholecystectomy to prevent postoperative cognitive dysfunction in elderly and senile patients.

\section{Materials and methods}

The study was conducted from 2017 to 2020. During the study conducted in the Municipal Non-profit Company (MNC) "O. I. Meshchaninov Memorial City Clinical Emergency Hospital” of the Kharkiv City Council, 84 patients diagnosed with gallstone disease (GSD), acute cholecystitis who underwent laparoscopic cholecystectomy (LCE) were examined. The average age was $68.1 \pm 0.63$ years, and the average duration of the operation was $61.1 \pm 2.7$ minutes.

Before the beginning of the study, according to the Declaration of Helsinki, all patients were informed about the purpose, methods and design of the study. They personally agreed in writing to participate in the study: clinical examination, collection of material for laboratory tests, filling out questionnaires.

The study approved by the Commission on Ethics and Bioethics as part of the Scientific and Methodological Council of Kharkiv Medical Academy of Postgraduate Education, according to protocol No. 1 of February 18, 2020.

The study used the following inclusion criteria:

1) age of patients from 60 to 90 years;

2) confirmed diagnosis of GSD, acute cholecystitis;

3) physical status according to the American Society of Anesthesiologists (ASA) classification - I-III;
4) coronary heart disease (CHD);
5) cardiosclerosis;
6) hypertension grade I-II;
7) heart failure stage I-IIA, grade I, II;
8) no cardiovascular events in the history (myocardial infarction, cerebral vascular accident).
The criteria for exclusion from the study were:
1) patient's age less than 60 or more than 90 years;
2) patient's physical status according to the American Society of Anesthesiologists (ASA) classification - IV; 
3) diabetes mellitus;

4) CNS diseases;

5) intracranial hypertension;

6) mental diseases;

7) the history of cardiovascular events (myocardial infarction, cerebral vascular accident);

8) patient's reluctance to participate in the study.

Based on the type of anesthesia, patients were randomized into 2 groups: Group I ( $n=42)-$ sevofluran (2-3 vol. \%) inhalation anesthesia with AVL; Group II ( $\mathrm{n}=42)-$ propofol ( $2 \mathrm{mg} / \mathrm{kg} / \mathrm{h})$ TIA with AVL. In both groups: analgesia - fentanyl $(1 \mathrm{mcg} / \mathrm{kg} / \mathrm{h})$, relaxation - atracurium (Table 1).

Table 1

Patient randomization based on the type of anesthesia

\begin{tabular}{cc} 
Group I (n=42) & Group II (n=42) \\
\hline sevofluran $(2-3$ vol. \%) inhalation anesthesia with AVL & propofol $(2 \mathrm{mg} / \mathrm{kg} / \mathrm{h})$ TIA with AVL \\
Analgesia - fentanyl $(1 \mathrm{mcg} / \mathrm{kg} / \mathrm{h})$, \\
Relaxation - atracurium
\end{tabular}

Monitoring of hemodynamic parameters consisted of determining heart rate (HR), pulse rate (PR), systolic blood pressure (SBP), diastolic blood pressure (DBP), mean arterial pressure (MAP), and cardiac output (CO). These indicators were measured using the "Vismo PVM-2701" NIHONKOHDEN monitor (Japan).

For continuous measurement and monitoring of functional oxygen saturation of cerebral hemoglobin $\left(\mathrm{rSO}_{2}\right)$, a non-invasive regional oximeter system Masimo O3 (USA) was used.

These indicators were continuously monitored throughout the entire period of anesthesia and surgery. However, for material processing the following stages were distinguished: stage 1 before premedication; stage 2 - after premedication; stage 3 - intubation; stage 4 - beginning of the operation; stage 5 - carbon dioxide insufflation; stage 6 - middle of the operation; stage 7 extubation; stage 8 - 20 min after the operation.

The patients' cognitive functions were assessed using neuropsychological scales: O.R. Luria's 10-word memory test and Mini-Mental State Examination (MMSE) scale. The memory evaluation according to $\mathrm{O}$. R. Luria uses a quantitative 10 -word memory test. The test is designed to diagnose auditory verbal memory.

The MMSE method is widely used in most modern epidemiological and clinical studies for screening and evaluating cognitive disorders, which confirms its validity and suitability. The MMSE scale consists of a number of sub-tests that allow to quickly and effectively evaluate time and spatial awareness, as well as short-term and long-term memory, speech function, gnosis, and praxis. The total score is calculated by adding up the scores for individual sub-tests.

The patients received cognitive evaluation three times: before the surgery, on the next day after the surgery, and on the fifth day after the surgery.

The obtained data were analyzed by statistical processing using SPSS19 software for Windows and descriptive statistics methods. Shapiro-Wilk test was used to determine the distribution of the obtained data. Provided the quantitative variables were in accordance with the normal distribution law, they were described as $\mathrm{M} \pm \mathrm{m}$. When studying the dynamics of indicators against the baseline levels in the case of normal distributions, Student's t-test was used for dependent samples, and for intergroup comparisons - Student's t-test was used for independent samples. The differences were considered statistically significant at $\mathrm{p}<0.05$.

\section{Results}

Patients did not differ significantly between the groups by hemodynamic parameters during LCE (Table 2).

It should be noted that patients in both groups showed an intraoperative decrease in hemodynamic parameters compared to the baseline, which is explained by the effect of general anes- 
thetics and opioids during general anesthesia. However, these changes were moderate and did not require correction. After the operation, hemodynamic parameters tended to increase, but remained slightly lower than the baseline.

Table 2

Comparative evaluation of hemodynamic parameters in patients at different stages of the study

\begin{tabular}{ccccccc}
\hline \multirow{2}{*}{ Stages of the study } & Group & \multicolumn{5}{c}{ Hemodynamic parameters } \\
\cline { 3 - 7 } Before premedication & I & $78.02 \pm 1.8$ & $157.98 \pm 2.1$ & $94.05 \pm 1.4$ & $114.95 \pm 1.4$ & $8.16 \pm 0.1$ \\
& II & $79.00 \pm 1.5$ & $153.93 \pm 2.4$ & $93.83 \pm 1.2$ & $113.52 \pm 1.4$ & $8.00 \pm 0.1$ \\
After premedication & I & $83.95 \pm 1.6$ & $143.81 \pm 1.7$ & $88.45 \pm 1.4$ & $106.55 \pm 1.4$ & $7.34 \pm 0.1$ \\
& II & $83.29 \pm 1.4$ & $141.31 \pm 1.8$ & $87.93 \pm 1.2$ & $105.43 \pm 1.2$ & $7.31 \pm 0.1$ \\
Intubation & I & $81.88 \pm 1.2$ & $135.83 \pm 1.5$ & $87.14 \pm 1.4$ & $103.24 \pm 1.3$ & $6.66 \pm 0.1$ \\
\multirow{2}{*}{ Beginning of the operation } & II & $78.81 \pm 1.2$ & $135.00 \pm 1.8$ & $84.29 \pm 1.4$ & $100.33 \pm 1.5$ & $6.61 \pm 0.1$ \\
\multirow{2}{*}{ CD insufflation } & I & $79.24 \pm 1.4$ & $127.38 \pm 1.8$ & $81.31 \pm 1.4$ & $96.60 \pm 1.5$ & $5.97 \pm 0.1$ \\
& II & $75.50 \pm 1.4$ & $129.05 \pm 1.9$ & $80.36 \pm 1.5$ & $96.33 \pm 1.6$ & $5.95 \pm 0.1$ \\
Middle of the operation & I & $79.12 \pm 1.4$ & $124.64 \pm 1.7$ & $78.88 \pm 1.4$ & $94.26 \pm 1.4$ & $5.48 \pm 0.1$ \\
& II & $76.12 \pm 1.4$ & $127.62 \pm 1.7$ & $79.88 \pm 1.4$ & $95.90 \pm 1.4$ & $5.57 \pm 0.1$ \\
End of the operation & I & $75.60 \pm 1.0$ & $132.74 \pm 1.3$ & $82.98 \pm 1.2$ & $99.07 \pm 1.1$ & $4.86 \pm 0.1$ \\
& II & $74.07 \pm 1.8$ & $135.60 \pm 1.5$ & $84.17 \pm 1.2$ & $101.10 \pm 1.2$ & $4.92 \pm 0.1$ \\
20 min after the operation & I & $75.33 \pm 1.3$ & $128.45 \pm 1.5$ & $82.02 \pm 2.2$ & $95.33 \pm 1.4$ & $6.73 \pm 0.04$
\end{tabular}

Note: *-statistically significant difference between the groups $(p<0.05)$

Table 3 shows changes in $\mathrm{rSO}_{2}$ during LCE in elderly and senile patients at various stages of the study.

Table 3

$\mathrm{rSO}_{2}$ dynamics in elderly and senile patients during laparoscopic cholecystectomy (\%)

\begin{tabular}{|c|c|c|c|c|c|c|}
\hline \multirow{2}{*}{ Stages of the study } & \multicolumn{3}{|c|}{ LH (left hemisphere of the brain) } & \multicolumn{3}{|c|}{ RH (right hemisphere of the brain) } \\
\hline & Group I & Group II & $\mathbf{p}$ & Group I & Group II & $\mathbf{p}$ \\
\hline Before premedication & $64.3 \pm 0.2$ & $64.5 \pm 0.2$ & $>0.05$ & $64.7 \pm 0.2$ & $64.8 \pm 0.2$ & $>0.05$ \\
\hline After premedication & $64.8 \pm 0.2$ & $64.4 \pm 0.2$ & $>0.05$ & $65.1 \pm 0.2$ & $64.8 \pm 0.2$ & $>0.05$ \\
\hline Intubation & $63.9 \pm 0.1$ & $64.1 \pm 0.2$ & $>0.05$ & $64.0 \pm 0.2$ & $64.2 \pm 0.2$ & $>0.05$ \\
\hline Beginning of the operation & $67.1 \pm 0.2$ & $66.1 \pm 0.2$ & $<0.001$ & $67.6 \pm 0.2$ & $66.6 \pm 0.2$ & $<0.001$ \\
\hline $\mathrm{CD}$ insufflation & $60.1 \pm 0.2$ & $60.0 \pm 0.2$ & $>0.05$ & $60.5 \pm 0.2$ & $60.3 \pm 0.2$ & $>0.05$ \\
\hline In the middle of the operation & $75.4 \pm 0.3$ & $72.9 \pm 0.3$ & $<0.001$ & $75.2 \pm 0.3$ & $73.3 \pm 0.4$ & $<0.001$ \\
\hline Extubation & $68.4 \pm 0.2$ & $68.1 \pm 0.2$ & $>0.05$ & $68.4 \pm 0.1$ & $67.9 \pm 0.2$ & $<0.05$ \\
\hline 20 min after the operation & $64.4 \pm 0.1$ & $64.2 \pm 0.2$ & $>0.05$ & $64.7 \pm 0.2$ & $64.6 \pm 0.1$ & $>0.05$ \\
\hline
\end{tabular}

The study results showed that $\mathrm{rSO}_{2}$ was significantly decreased during the $\mathrm{CD}$ insufflation as compared to before premedication: in LH - by $7.0 \%(\mathrm{p}<0.001)$, in $\mathrm{RH}-$ by $6.9 \%(\mathrm{p}<0.001)$ (Group I); in LH - by $7.4 \%(p<0.001)$, in $\mathrm{RH}-$ by $7.5 \%(\mathrm{p}<0.001)$ (Group II). The difference in $\mathrm{rSO}_{2}$ between the groups during the operation is also significant, which may evidence that sevoflu- 
rane preserves $\mathrm{rSO}_{2}$ somewhat better than propofol: in Group I, $\mathrm{rSO}_{2}$ was significantly increased in the middle of the operation as compared to the period before premedication: in LH - by $14.66 \%$ $(\mathrm{p}<0.001)$, in RH - by $13.94 \%(\mathrm{p}<0.001)$; in Group II, in LH - by $11.60 \%(\mathrm{p}<0.001)$, in RH - by $11.53 \%(\mathrm{p}<0.001)$.

In addition, the dynamic pattern of the results of the cognitive tests was studied.

In Group I, the results of Luria's test significantly decreased the day after the operation, as compared to before premedication, by $8.7 \%(\mathrm{p}<0.001)$, insignificantly decreased on the fifth day after the operation, as compared to before premedication, by $2.7 \%$ ( $>>0.05)$. In Group II, the test results significantly decreased the day after the operation, as compared to before premedication, by $10.1 \%(\mathrm{p}<0.001)$, insignificantly decreased on the fifth day after the operation, as compared to before premedication, by $2.7 \%(\mathrm{p}>0.05)$.

In Group I, the results of MMSE test significantly decreased the day after the operation, as compared to before premedication, by $6.0 \%(\mathrm{p}<0.001)$, insignificantly decreased on the fifth day after the operation, as compared to before premedication, by $0.35 \%(p>0.05)$. In Group II, the results significantly decreased the day after the operation, as compared to before premedication, by $6.3 \%(p<0.001)$, insignificantly decreased on the fifth day after the operation, as compared to before premedication, by $0.35 \%(\mathrm{p}>0.05)$.

\section{Discussion}

At the moment, the assessment of postoperative cognitive dysfunction is not a standard component for assessing the quality of anesthesia, although, in our opinion, the quality of life of patients depends directly on the level of cognitive functioning of the patient [10].

Some researchers note that general anesthesia is associated with a more significant decrease in $\mathrm{rSO}_{2}$ and point to a relationship between the occurrence of POCD and $\mathrm{rSO}_{2}$ value, when an intraoperative decrease in $\mathrm{rSO}_{2}$ is a predictor of POCD. X. Li et al. [11] showed this relationship in thoracic surgery, G. Papadopoulos et al. [12] - in operations for hip fractures in patients over 75 years of age. Some randomized studies have shown the use of oximetry to minimize brain destruction, based on the fact that a decrease in $\mathrm{rSO}_{2}$ is associated with impaired cognitive function after surgery [13]. The use of cerebral oximetry is proposed not only to identify the risk of developing POCD, but also to determine the tactics of postoperative management of patients. Some researchers also report the relationship between cerebral oximetry values and the use of specific anesthetics [14] with the premorbid POCD background [15]. Thus, T. Ružman et al. [16] compared the effect on regional cerebral oxygenation $\left(\mathrm{rSO}_{2}\right)$ during inhalation anesthesia with sevoflurane and total intravenous anesthesia with propofol during LCE and concluded that sevoflurane anesthesia provides 4-11\% higher $\mathrm{rSO}_{2}$ values compared to propofol and significantly fewer critical $\mathrm{rSO}_{2}$ decreases in LCE. D. Salazar et al. [17] suggested that development of POCD was associated with a decrease in $\mathrm{rSO}_{2}$ at a certain patient's position during the operation, and although a reliable relationship could not be identified, the authors concluded that protocols with $\mathrm{rSO}_{2}$ measurement reduce the risk of POCD development. Other researchers have noted a decrease in $\mathrm{rSO}_{2}$ when the patient is in the beach chair position [18]. In addition, the severity and frequency of POCD were reported to be decreased with a decrease in the level of $\mathrm{rSO}_{2}$ when using a certain algorithm of actions, but the effect on the incidence of POCD is not the same in various studies [19]. This may be due either to the fact that measures aimed at preventing a decrease in $\mathrm{rSO}_{2}$ levels in the brain are insufficient, or, conversely, the relationship between $\mathrm{rSO}_{2}$ values and POCD is weak. F. Holmgaard et al. [20] found no differences in intraoperative $\mathrm{rSO}_{2}$ values in patients with and without POCD either at discharge or 3 months after a cardiac surgery.

According to our study, the parameters of cerebral oxygenation $\mathrm{rSO}_{2}$ decreased at carbon dioxide insufflation, but this decrease was short-term and lasted no more than 5 minutes. The decrease in $\mathrm{rSO}_{2}$ could occur due to carbon dioxide insufflation, which is significantly associated with the potentiation of intracranial hypertension due to increased intra-abdominal pressure, delayed venous outflow from the brain and slow cerebral circulation. In its turn, the position of Trendelenburg can also cause an increase in intra-abdominal pressure and a decrease in $\mathrm{rSO}_{2}$. Intraoperative monitoring of cerebral oxygenation using regional cerebral oximetry allowed to reveal a 
short-term decrease in $\mathrm{rSO}_{2}$ and take timely measures to prevent further decrease in $\mathrm{rSO}_{2}$ and development of stable POCD. A single-factor analysis of variance did not reveal any significant effect of episodic decrease in $\mathrm{rSO}_{2}$ during the carbon dioxide insufflation on the decrease in cognitive functions in the postoperative period.

However, the issue of using regional cerebral oximetry in all elderly and senile patients during LCE remains relevant.

Research limitations. The study was not performed in case of neurological history or mental disorder of the patient, as well as in case of refusal of the patient to participate in the study.

Prospects for further researches. The study does not exhaust the entire depth of the problem of preventing the appearance of postoperative cognitive dysfunctions in elderly patients. In this regard, further work in this direction is possible. Also, the sample size was quite small, and statistical reliability was insufficient. Therefore, future studies require sampling with more patients.

\section{Conclusions}

During LCE, elderly and senile patients showed a short-term decrease in $\mathrm{rSO}_{2}$ at the stage of carbon dioxide insufflation. $\mathrm{rSO}_{2}$ was found to be significantly decreased during the carbon dioxide insufflation as compared to before premedication: in LH - by $7.0 \%$, in RH - by $6.9 \%$ (Group I); in $\mathrm{LH}$ - by $7.4 \%$, in $\mathrm{RH}$ - by $7.5 \%$ (Group II). But there was no significant effect on the temporary decrease in cognitive functions in the early postoperative period. The day following the surgery, cognitive functions significantly decreased by $8.7 \%$ on the Luria's test, by $6.0 \%$ on the MMSE test (Group I); in Group II - by $10.1 \%$ and $6.3 \%$, respectively, as compared to before premedication. On Day 5 after the surgery, cognitive functions decreased by $2.7 \%$ on the Luria's test, by $0.35 \%$ on the MMSE test (Group I); in Group II - by $2.7 \%$ and $0.35 \%$, respectively, as compared to before premedication.

A decrease in local brain tissue oxygenation $\left(\mathrm{rSO}_{2}\right)$ can occur during LCE in the Trendelenburg position, despite the fact that other intraoperative indicators remain stable, which allows it to remain unrecognized.

Non-invasive intraoperative regional cerebral oximetry makes it possible to quickly assess the $\mathrm{rSO}_{2}$ value and identify possible episodes of its decline as early as possible.

The use of non-invasive intraoperative regional cerebral oximetry makes it possible to take timely measures to correct $\mathrm{rSO}_{2}$ values and prevent brain hypoxia events.

Non-invasive regional cerebral oximetry may help reduce postoperative cognitive dysfunction.

Non-invasive $\mathrm{rSO}_{2}$ monitoring in the perioperative period contributes to alertness and timely measures to prevent postoperative cognitive dysfunction.

\section{Conflict of interests}

The authors declare that they have no conflicts of interest.

\section{References}

[1] Strøm, C., Rasmussen, L. S., Sieber, F. E. (2013). Should general anaesthesia be avoided in the elderly? Anaesthesia, $69,35-44$. doi: http://doi.org/10.1111/anae.12493

[2] Vutskits, L., Xie, Z. (2016). Lasting impact of general anaesthesia on the brain: mechanisms and relevance. Nature Reviews Neuroscience, 17 (11), 705-717. doi: http://doi.org/10.1038/nrn.2016.128

[3] Wu, L., Zhao, H., Weng, H., Ma, D. (2019). Lasting effects of general anesthetics on the brain in the young and elderly: "mixed picture" of neurotoxicity, neuroprotection and cognitive impairment. Journal of Anesthesia, 33 (2), 321-335. doi: http:// doi.org/10.1007/s00540-019-02623-7

[4] Végh, T. (2016). Cerebral Oximetry in General Anaesthesia. Turkish Journal of Anesthesia and Reanimation, 44 (5), $247-249$. doi: http://doi.org/10.5152/tjar.2016.26092016

[5] Green, D. W., Kunst, G. (2017). Cerebral oximetry and its role in adult cardiac, non-cardiac surgery and resuscitation from cardiac arrest. Anaesthesia, 72, 48-57. doi: http://doi.org/10.1111/anae.13740 
[6] Grocott, H. P. (2017). Advancing Cerebral Oximetry Research One Small Step at a Time. Journal of Cardiothoracic and Vascular Anesthesia, 31 (4), 1151-1152. doi: http://doi.org/10.1053/j.jvca.2017.03.030

[7] Li, X., Shao, M., Wang, J., Wang, Y. (2014). Relationship between post-operative cognitive dysfunction and regional cerebral oxygen saturation and $\beta$-amyloid protein. Journal of Zhejiang University SCIENCE B, 15 (10), 870-878. doi: http:// doi.org/10.1631/jzus.b1400130

[8] Badenes, R., García-Pérez, M. L., Bilotta, F. (2016). Intraoperative monitoring of cerebral oximetry and depth of anaesthesia during neuroanesthesia procedures. Current Opinion in Anaesthesiology, 29 (5), 576-581. doi: http://doi.org/10.1097/ aco.0000000000000371

[9] Mashour, G. A., Woodrum, D. T., Avidan, M. S. (2015). Neurological complications of surgery and anaesthesia. British Journal of Anaesthesia, 114 (2), 194-203. doi: http://doi.org/10.1093/bja/aeu296

[10] Li, X., Li, F., Liu, Z., Shao, M. (2015). Investigation of one-lung ventilation postoperative cognitive dysfunction and regional cerebral oxygen saturation relations. Journal of Zhejiang University-SCIENCE B, 16 (12), 1042-1048. doi: http:// doi.org/10.1631/jzus.b1500030

[11] Papadopoulos, G., Karanikolas, M., Liarmakopoulou, A., Papathanakos, G., Korre, M., Beris, A. (2012). Cerebral Oximetry and Cognitive Dysfunction in Elderly Patients Undergoing Surgery for Hip Fractures: A Prospective Observational Study. The Open Orthopaedics Journal, 6 (1), 400-405. doi: http://doi.org/10.2174/1874325001206010400

[12] Denault, A., Deschamps, A., Murkin, J. M. (2007). A Proposed Algorithm for the Intraoperative Use of Cerebral Near-Infrared Spectroscopy. SeminarsinCardiothoracic and VascularAnesthesia, 11 (4),274-281. doi:http://doi.org/10.1177/1089253207311685

[13] Choi, J. W., Joo Ahn, H., Yang, M., Kim, J. A., Lee, S. M., Ahn, J. H. (2015). Comparison Between Phenylephrine and Dopamine in Maintaining Cerebral Oxygen Saturation in Thoracic Surgery. Medicine, 94 (49), e2212. doi: http://doi.org/10.1097/ md.0000000000002212

[14] Kim, S. Y., Chae, D. W., Chun, Y.-M., Jeong, K. H., Park, K., Han, D. W. (2016). Modelling of the Effect of End-Tidal Carbon Dioxide on Cerebral Oxygen Saturation in Beach Chair Position under General Anaesthesia. Basic \& Clinical Pharmacology \& Toxicology, 119 (1), 85-92. doi: http://doi.org/10.1111/bcpt.12549

[15] Ružman, T., Šimurina, T., Gulam, D., Ružman, N., Miškulin, M. (2017). Sevoflurane preserves regional cerebral oxygen saturation better than propofol: Randomized controlled trial. Journal of Clinical Anesthesia, 36, 110-117. doi: http:// doi.org/10.1016/j.jclinane.2016.10.010

[16] Salazar, D., Sears, B. W., Aghdasi, B., Only, A., Francois, A., Tonino, P., Marra, G. (2013). Cerebral desaturation events during shoulder arthroscopy in the beach chair position: patient risk factors and neurocognitive effects. Journal of Shoulder and Elbow Surgery, 22 (9), 1228-1235. doi: http://doi.org/10.1016/j.jse.2012.12.036

[17] Yu, Y., Zhang, K., Zhang, L., Zong, H., Meng, L., Han, R. (2018). Cerebral near-infrared spectroscopy (NIRS) for perioperative monitoring of brain oxygenation in children and adults. Cochrane Database of Systematic Reviews. doi: http://doi.org/ 10.1002/14651858.cd010947.pub2

[18] Zorrilla-Vaca, A., Healy, R., Grant, M. C., Joshi, B., Rivera-Lara, L., Brown, C., Mirski, M. A. (2018). Intraoperative cerebral oximetry-based management for optimizing perioperative outcomes: a meta-analysis of randomized controlled trials. Canadian Journal of Anesthesia/Journal Canadien D’anesthésie, 65 (5), 529-542. doi: http://doi.org/10.1007/s12630-018-1065-7

[19] Holmgaard, F., Vedel, A. G., Rasmussen, L. S., Paulson, O. B., Nilsson, J. C., Ravn, H. B. (2019). The association between postoperative cognitive dysfunction and cerebral oximetry during cardiac surgery: a secondary analysis of a randomised trial. British Journal of Anaesthesia, 123 (2), 196-205. doi: http://doi.org/10.1016/j.bja.2019.03.045 